\title{
Die „Hot Topics“ der interventionellen Radiologie
}

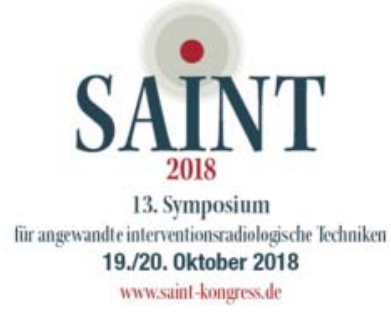

Revolutionäre Ideen für die radiologische Therapie von Prostataerkrankungen und „state of the art"-Fortbildungen: Das 13. Symposium für angewandte interventionsradiologische Techniken SAINT 2018 bietet ein abwechslungsreiches Programm für Newcomer und Chefärzte. Im Interview erzählen die wissenschaftlichen Leiter, Prof. Dr. Markus Düx vom Krankenhaus Nordwest in Frankfurt und Professor Prof. Dr. Christian Stroszczynski vom Universitätsklinikum Regensburg, welche Schwerpunktthemen die Teilnehmer erwarten und welche Vorteile das fast schon familiäre Ambiente bietet.

Herr Professor Düx, Herr Professor Stroszczynski, was zeichnet den SAINT 2018 aus und welche Schwerpunktthemen haben Sie gesetzt?

Prof. Dr. Markus Düx Wir werden auch dieses Jahr einen sehr attraktiven SAINT anbieten. Neue Veranstaltungsformate ergänzen das besondere persönliche Ambiente der Veranstaltung, das wir gerne erhalten möchten. Mit Professor Stroszczynski, der den SAINT seit der Geburtsstunde kennt und unterstützt, habe ich den richtigen Partner gefunden, die Veranstaltung breiter aufzustellen.

Prof. Dr. Christian Stroszczynski Wir hoffen, mit dem SAINT eine kompakte Übersicht über den aktuellen Stand der interventionellen Radiologie insgesamt zu bieten. Daher sind die Themen „Arterielle Intervention“ und „Tumortherapie“ gesetzt. Darüber hinaus werden dieses Jahr die Prostata und die Navigation als "Hot Topics“ behandelt. In der Navigation widmen wir uns beispielsweise der MRT-Ultraschall-Fusionsbiopsie und der Stereotaxie und schauen uns genauer an, wie weit die Robotik in der Intervention gekommen ist. In Bezug auf das Prostatakarzinom werfen wir u. a. einen Blick auf die S3-Leitlinie.

Wieso haben Sie gerade diese Bereiche ausgesucht - gibt es hier einen besonderen Fortbildungsbedarf oder spannende neue Entwicklungen?

Stroszczynski Die interventionelle Radiologie ist ein sehr dynamisches Fach, das sich an jedem Standort anders entwickelt. Deshalb war es uns wichtig, dass wir einen wissenschaftlichen Beirat gründen, der stets die „Hot Topics“ des Jahres aufspürt. Zusammen mit Professor Frank Wacker von der Medizinischen Hochschule Hannover und Professor Philipp Wiggermann vom Klinikum Braunschweig waren wir uns einig, dass wir die radiologischen Therapien des Prostataadenoms und des Prostatakarzinoms sowie die Navigation aufnehmen müssen. Die Prostata-Arterienembolisation und perkutane Ablation haben das Potenzial, die Prostatatherapie flächendeckend zu revolutionieren - das Interesse der Patienten ist immens. Hinsichtlich der Navigation sind neue pfiffige Produkte entwickelt worden, die auf den Markt drängen.

Herr Professor Stroszczynski, Sie sind erstmals gemeinsam mit Professor Düx wissenschaftlicher Leiter des SAINT. Welche Themen sind Ihnen besonders wichtig?

Stroszczynski Ziel ist es, den Charakter der etablierten Veranstaltung zu bewahren. Die deutsche Interventionsszene trifft sich seit über einem Jahrzehnt nicht ohne Grund so gerne im Rheingau, da es Professor Düx gelungen ist, die richtige Mischung zu finden. Auf dem SAINT bekommt jeder Teilnehmer, ob Chefarzt, Newcomer oder Industriepartner, ohne viel Ablenkung eine kompakte Übersicht über die neusten technischen und klinischen Entwicklungen in der interventionellen Radiologie, dazu DeGIR-Weiterbildungsinhalte zum Neuerwerb oder zur Auffrischung. Als gebürtiger Wiesbadener weiß ich zudem, dass das Rahmenprogramm im Rheingau schwer zu toppen ist.

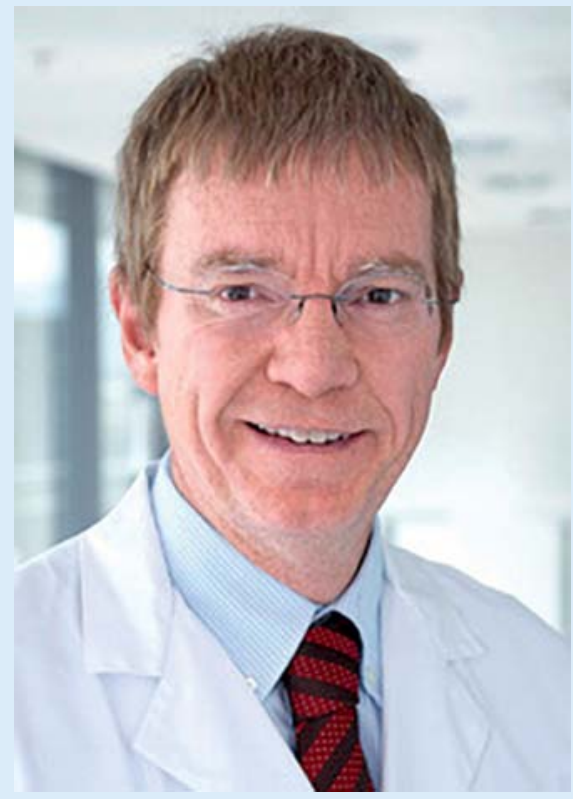

Prof. Dr. Markus Düx

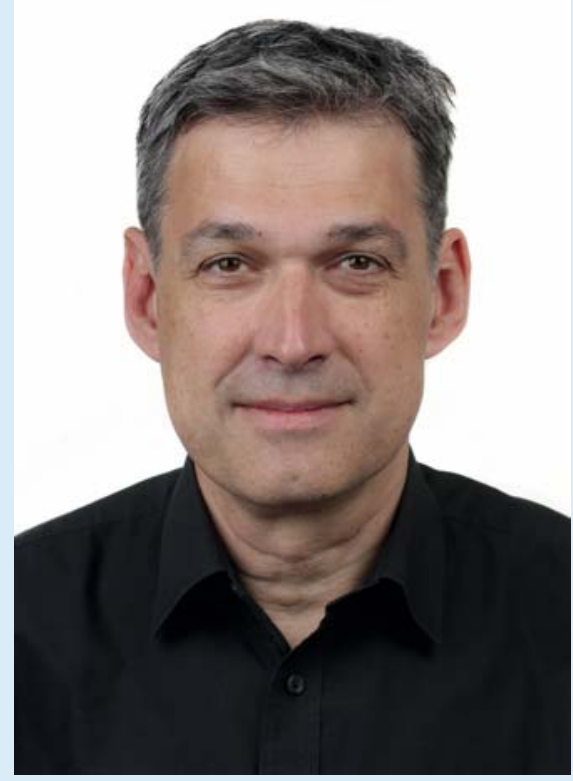

Prof. Dr. Christian Stroszczynski

Welche Veranstaltungsformate erwarten die Teilnehmerinnen und Teilnehmer?

Düx Wir setzen auf eine Trias: Die beiden Hauptthemen „Arterielle Intervention“ und „Tumortherapie“ widmen sich dem „state of the art“ im Rahmen von DeGIR-Zertifizierungen und Fortbildungssessions. Hier wird 
fallbezogen diskutiert, mit Zeit für Austausch und interaktive TED-Formate. Neue Medizinprodukte werden in den Interventionsküchen vorgestellt und unsere Hot-Topics „Prostata“ und „Navigation“ präsentieren wir in Fortbildungsvorträgen mit viel Raum für Fragen und Diskussionen.

An wen richtet sich das Symposium? Gibt es auch Sessions für den radiologischen Nachwuchs?

Stroszczynski Aufgrund der zentralen Lage bei Frankfurt ist der SAINT für alle Interventionsradiologen aus ganz Deutschland gut erreichbar. Für die Newcomer sind vor allem die fallbasierten Veranstaltungen klasse, ebenso die Sitzung „Morgen habe ich zum ersten Mal Interventionshintergrund“. Darüber hinaus ist der SAINT ein idealer Platz für Networking in der interventionellen Radiologie. Im Kontrast zu den eher anonymen Großveranstaltungen kann man hier praktische Tipps zur Intervention einholen, aber auch wertvolle Kontakte mit etablierten interventionellen Radiologen knüpfen. Noch dazu gibt es für Einsteiger einen erheblichen Preisrabatt.

Herr Professor Düx, Sie haben dieses etablierte Symposium ins Leben gerufen. Wie möchten Sie den SAINT künftig weiterentwickeln?

Düx Ich habe den SAINT zum ersten Mal 2006 durchgeführt. Einen inhaltlich hochwertigen interventionell-radiologischen Weiterbildungskongress in einem familiären Ambiente anzubieten, hat sich etabliert. Aber wir müssen auch mit der Zeit gehen. Unser SAINT muss sich öffnen für neue Formate, so zum Beispiel für die strukturierten Weiterbildungsmodule in der interventionellen Radiologie, die die DeGIR in den letzten Jahren entwickelt hat. Also war es für mich nur logisch, den Schulterschluss mit der DRG und der DeGIR zu suchen, um vor allem auch für junge Kollegen, die wir an die interventionelle Radiologie heranführen wollen, ein noch besseres Forum zu bieten. Hier spielt der bereits erwähnte Kongressbeirat mit Professor Wacker und
Professor Wiggermann eine wichtige Rolle. Ihre Unterstützung zahlt sich aus, denn jeder von uns hat eine etwas andere Blickrichtung auf die interventionelle Radiologie. Ich kann nur sagen: Kommen Sie zum SAINT! Sie werden interventionelle Radiologie auf höchstem Niveau geboten bekommen und mit renommierten Experten diskutieren können! Dazu passt der besondere Spirit des SAINT in der wunderbaren Umgebung des Schlosses Johannisberg, das inmitten einer der schönsten Weinkulturlandschaften Deutschlands liegt.

\section{SAINT 2018}

13. Symposium für angewandte interventionsradiologische Techniken

19. und 20. Oktober 2018

Schloss Johannisberg/Geisheim im Rheingau

https://www.saint-kongress.de 